\title{
EFEITOS DO TRATAMENTO DE SEMENTES DE ALGODÃO (Gossypium hirsutum L.), ARROZ (Oryza sativa L.) E SOJA [Glycine max (L.) MERRIL] COM ALGUNS FUNGICIDAS NÃO MERCURIAIS*
}

\author{
JULIO MARCOS FILHO** \\ ANTONIO CARLOS FERRAZ ***
}

\section{RESUMO}

Esta pesquisa foi conduzida com o objetivo de estudar o comportamento de sementes de arroz, algodão e soja, quando tratadas com fungicidas não mercuriais, através de testes de germinação e de vigor.

Foram utilizados os seguintes produt ss: Arasan (Thiram $75 \%$ i.a.), Panoctine (Guazatine $75 \%$ i.a.) e Terracoat $(23,2 \%$ PCNB + 5,8\% Terrazole). A dosagem do 19 produto foi de $100 \mathrm{~g} / 100 \mathrm{~kg}$ de sementes enquanto que, para os outros dois, foram de 200,400 e $600 \mathrm{~cm}^{3} / 100 \mathrm{~kg}$.

Nas condições do experimento, as três dosagens de Panoctine foram tóxicas para sementes de arroz e a dose mais alta prejudicou o vigor de soja e de algodão.

Por outro lado Arasan e Terracoat não prejudicaram a germinação e vigor de arroz e de soja e apresentarm efeitos benéficos sobre o vigor de sementes de algodão.

\section{INTRODUÇÃO}

O tratamento de sementes com fungicidas possibilita o controle de inümeras moléstias transmitidas por sementes; oferece, ainda, proteção às sementes e às plântulas contra a ação de microrganismos presentes no solo e contra os efeitos desfavoráveis do ambiente, após a semeadura ou durante o armazenamento.

Os fungicidas organo-mercuriais embora muito eficientes, apresentam problemas devido a suas características residuais e tóxicas e sua utilização é proibida em inúmeros países (PULIDO, 1972).

Desta maneira foi planejado o presente trabalho, com o objetivo de testar a influência de alguns compostos não mercuiriais sobre a germinação e o vigor de sementes de algodão, de arroz e de soja, pois além da eficiência no controle às moléstias, um fungicida empregado para o tratamento de sementes não deve afetar a qualidade das mesmas.

\footnotetext{
* Entregue para publicação em 11/12/1975.

** Prof. Assist. Doutor - Departamento de Agricultura e Horticultura, ESALQ/USP.

** Bolsista do Departamento de Agricultura e Horticultura, ESALQ/USP.
} 


\section{REVISÃO DA LITERATURA}

As informações disponiveis sobre a influência do tratamento fungicida sobre a germinação e o vigor de sementes, embora importantes e necessárias, são relativamente escassas. A maioria das pesquisas sobre o assunto referem-se principalmente ao emprego de fungicidas mercuriais, para o controle de moléstias transmitidas por sementes ou provocadas por microrganismos presentes no solo, sem considerar os possíveis efeitos fitotóxicos dos produtos utilizados.

Segundo CHRISTIDIS (1947) o tratamento de sementes de algodão geralmente é eficiente e vantajoso, principalmente quando as condições são favoráveis à ocorrência de moléstias. Ressaltou, entretanto, que os resultados são muito influenciados pela variedade, características da semente e pelo ambiente.

Trabalhando com sementes de soja, ATHOW \& CALDWELL (1956) verificaram que o tratamento com Arasan $(200 \mathrm{~g} / 100 \mathrm{~kg}$ ) beneficiou a porcentagem de emergência das plântulas apenas quando as sementes tratadas eram de baixa qualidade; os efeitos do produto em sementes sadias foram pouco acentuados.

De acordo com BRITTON (1960), o emprego de fungicidas em sementes de soja é justificado quando a porcentagem de germinação é inferior a $80 \%$; dentre os produtos testados, os melhores resultados foram obtidos com a aplicação de Arasan.

Por outro lado, MELLO \& ROSSETTI (1960) verificaram que Granosan M (90 g/ $/ 100 \mathrm{~kg}$ ) foi superior a outros produtos pesquisados para o tratamento de sementes de arroz.

ABRAHÃO \& ANDRȦDE (1961) estudaram a influência de fungicidas sobre a germinação e a produção de algodão. Observaram que o comportamento de sementes tratadas com PCNB, nas dosagens de 300 e $600 \mathrm{~g} / 100 \mathrm{~kg}$, foi semelhante ao da testemunha; o tratamento com Arasan $(200 \mathrm{~g} / 100 \mathrm{~kg})$, por sua vez, originou menor germinação que a testemunha. As diferenças entre os efeitos dos fungicidas e a testemunha foram devidos à ocorrência de moléstias e não à fitotoxicidade dos produtos.

PEREIRA GONÇALO (1963) tratou sementes de soja com Arasan e Neantina, tendo observado que os dois fungicidas protegeram a germinação de sementes de baixa qualidade. Quando o material era de qualidade superior, as diferenças com a testemunha foram pequenas.

ABRAHÃO e colaboradores (1964), verificaram que o tratamento de sementes de algodão com a mistura entre PCNB e Arasan foi tão eficiente quanto à aplicaçâo de fungicidas mercuriais para a proteção da germinação. Segundo estes autores, a mistura apresenta a vantagem de ser menos fitotóxica.

Procurando determinar a eficiência de diferentes fungicidas aplicados em sementes de arroz, OLIVEIRA \& FALCÃO (1970) realizaram diversos testes de germinação e observaram que Neantina foi superior dos demais tratamentos e à testemunha, com exceção para Arasan; este produto não diferiu da testemunha.

TOLEDO e outros (1971), por sua vez, concluíram que sementes de arroz tratadas com Neantina $(200 \mathrm{~g} / 100 \mathrm{~kg})$, Brassicol $(300 \mathrm{~g} / 100 \mathrm{~kg})$ e Vitavax $(300 \mathrm{~g} / 100 \mathrm{~kg})$ apre- 
sentaram porcentagem de germinação superior à da testemunha, em dois experimentos conduzidos.

NETO e colaboradores (1975) estudaram a influência do tratamento com fungicidas, dentre os quais Panoctine $\left(200 \mathrm{~cm}^{3} / 100 \mathrm{~kg}\right)$, Rhodiauram $(200 \mathrm{~g} / 100 \mathrm{~kg})$ e Terracoat $\left(200 \mathrm{~cm}^{3} / 100 \mathrm{~kg}\right)$ sobre a germinação de sementes de soja. Nos testes instalados logo após e 30 dias após os tratamentos, todos os fungicidas, com exceção de Rhodiauram, prejudicaram o poder germinativo; aos 240 dias de armazenamento, verificaram-se efeitos benéficos de Rhodiauram e efeitos depressivos de Panoctine.

Segundo DELOUCHE \& CALDWELL (1960), o teste padrão de germinação muitas vezes não acusa diferenças de qualidade entre lotes de sementes porque um dos objetivos principais é a obtenção da máxima porcentagem de germinação que uma amostra pode oferecer; para tanto é conduzido sob condições artificiais muito favoráveis. Esses autores recomendaram a utilizaçăo de testes de vigor em pesquisas com sementes, para que sejam reveladas diferenças não detectadas pelo teste de germinação.

\section{MATERIAIS E MÉTODOS}

Foram empregadas para o presente estudo, sementes de algodáo, de arroz e de soja. As de algodão, variedade IAC 13-1 e as de arroz, variedade IAC 1246, foram obtidas junto ao Posto de Sementes da Divisão Regional Agrícola, em Campinas, SP, enquanto as de soja Santa Rosa, junto a Sementes Jaboticabal S/A, Jaboticabal, SP.

As sementes de algodão foram deslintadas quimicamente com ácido sulfúrico concentrado, na proporção de 1 parte de ácido : 4 de sementes.

Posteriormente, as sementes foram submetidas a escolha cuidadosa, resultando lotes constituídos, exclusivamente, de sementes fisicamente puras, que foram armazenadas em câmara seca do Laboratório de Sementes do Departamento de Agricultura e Horticultura, ESALQ/USP, para sua conservação e uniformização do teor de umidade.

Os três lotes permaneceram armazenados em câmara secâ durante um ano, sendo retiradas periodicamente apenas as amostras destinadas aos tratamentos fungicidas.

Por ocasião da instalação dos testes, retirou-se oito amostras de cada um dos lotes, que foram tratadas com diferentes fungicidas, de acordo com as especificações que se encontram no Quadro 1. 
QUADRO 1 - Tratamentos fungicidas: nomes comerciais, nomes técnicos e doses utilizadas.

\begin{tabular}{clll}
\hline Tratamento & Nome Comercial & $\begin{array}{c}\text { Nome Técnico } \\
\text { e concentração }\end{array}$ & $\begin{array}{c}\text { Dosagem/100 kg } \\
\text { de sementes }\end{array}$ \\
\hline$T_{0}$ & Testemunha & Thiram (75\% i.a.) & $100 \mathrm{~g}$ \\
$\mathrm{~T}_{1}$ & Arasan & Guazatine $(35 \%$ i.a.) & $200 \mathrm{~cm}^{3}$ \\
$\mathrm{~T}_{2}$ & Panoctine & Guazatine $(35 \%$ i.a.) & $400 \mathrm{~cm}^{3}$ \\
$\mathrm{~T}_{3}$ & Panoctine & Guazatine $(35 \%$ i.a.) & $600 \mathrm{~cm}^{3}$ \\
$\mathrm{~T}_{4}$ & Panoctine & PCNB $(23,2 \%)+$ Terrazole $(5,8 \%)$ & $200 \mathrm{~cm}^{3}$ \\
$\mathrm{~T}_{5}$ & Terracoat & PCNB $(23,2 \%)+$ Terrazole $(5,8 \%)$ & $400 \mathrm{~cm}^{3}$ \\
$\mathrm{~T}_{6}$ & Terracoat & PCNB $(23,2 \%)+$ Terrazole $(5,8 \%)$ & $600 \mathrm{~cm}^{3}$ \\
$\mathrm{~T}_{7}$ & Terracoat &
\end{tabular}

Para se efetuar o tratamento na forma líquida (Panoctine e Terracoat), foi empregado um pulverizador manual, tipo toalete, no interior do qual era colocado um tubo de ensaio graduado, com divisões de $1 \mathrm{~cm}^{3}$. As dosagens de 200,400 e $600 \mathrm{~cm}^{3} / 100 \mathrm{~kg}$ de sementes, foram obtidas através de aplicações na base de $2 \mathrm{~cm}^{3} / \mathrm{kg}$ de sementes, procedendo-se sempre a uma cuidadosa homogeinização entre cada uma das pulverizações.

A aplicação do fungicida Arasan, na forma de pó seco, foi efetuada em um recipiente metálico, no qual era colocado $1 \mathrm{~kg}$ de sementes e o produto na dosagem adequada; após fechado, o recipiente era movimentado manualmente.

Posteriormente, eram retiradas porçōes de cada um dos tratamentos, para a instalação de testes de germinação e de vigor.

Os tratamentos fungicidas, os testes de germinação e de vigor com as sementes de arroz e de soja foram efetuados em cinco épocas diferentes, a saber: julho, setembro e novembre de 1974 e fevereiro e abril de 1975. Essas épocas serão designadas no presente trabalho por $E_{1}, E_{2}, E_{3}, E_{4}$ e $E_{5}$, respectivamente.

Os tratamentos e os testes com sementes de algodão foram efetuados apenas nas quatro primeiras épocas.

Nas análises de germinação seguiu-se o procedimento das Regras para Análise de Sementes (MINISTÉRIO DA AGRICULTURA, 1967), com a modificação de se utilizar quatro repetições de cinqüenta sementes por tratamento. Foi empregado papel-toalha XUGA como substrato e as sementes foram colocadas para germinar em um aparelho STULTS a $30^{\circ} \mathrm{C}$.

$\mathrm{O}$ vigor das sementes foi avaliado pelo método do envelhecimento rápido, em câmara DE LEO, a $420 \mathrm{C}$ e $100 \%$ de umidade relativa. Para tanto, eram colocadas nia câmara previamente preparada, as sementes representativas dos oito tratamentos de cada espécie.

As sementes de soja e de algodão permaneciam no interior da câmara durante 48 horas; encerrado esse prazo eram retiradas para dar lugar às de arroz que ai permaneciam durante 120 horas. Em seqüência, eram colocadas para germinar em aparelho 
STULTS a 300C. As contagens do número de plântulas normais foram efetuadas no quarto dia após a colocação das sementes no germinador.

Os dados de germinação e de vigor foram transformados em arc sen $\sqrt{\%}$ e submetidos à análise estatística, separadamente para cada espécie e cada época, de acordo com esquema inteiramente casualizado. As médias foram comparadas pelo método de Tukey.

\section{RESULTADOS E DISCUSSÃO}

1. Algodão: Germinação e Vigor - Nos Quadros 2 e 3 encontram-se as médias obtidas para os efeitos dos tratamentos sobre a germinação e vigor, nas quatro épocas estudadas.

QUADRO 2 - Germinação de sementes de algodão: médias obtidas para os efeitos de tratamentos $(x=\operatorname{arcsen} \sqrt{\%})$.

\begin{tabular}{ccccc}
\hline Tratamentos & & & Épocas & \\
\hline & $\mathrm{E}_{1}$ & $\mathrm{E}_{2}$ & $\mathrm{E}_{3}$ & $\mathrm{E}_{4}$ \\
\hline $\mathrm{T}_{0}$ & 60,66 & 74,79 & 65,63 & 54,17 \\
$\mathrm{~T}_{1}$ & 69,52 & 79,50 & 77,23 & 71,09 \\
$\mathrm{~T}_{2}$ & 75,38 & 79,79 & 74,53 & 57,17 \\
$\mathrm{~T}_{3}$ & 67,04 & 73,83 & 73,23 & 65,75 \\
$\mathrm{~T}_{4}$ & 64,98 & 72,87 & 71,59 & 62,86 \\
$\mathrm{~T}_{5}$ & 71,10 & 78,94 & 67,41 & 68,79 \\
$\mathrm{~T}_{6}$ & 72,40 & 75,81 & 76,93 & 68,98 \\
$\mathrm{~T}_{7}$ & 71,28 & 78,28 & 73,31 & 61,12 \\
\hline d.m.s. $-5 \%$ (Tukey) & 14,00 & 10,89 & 14,51 & 14,88 \\
\hline C.V. (\%) & 8,67 & 6,07 & 8,56 & 9,98 \\
\hline
\end{tabular}


QUADRO 3 - Vigor de sementes de algodão: médias obtidas para os efeitos de tratamentos $(x=\operatorname{arcsen} \sqrt{\%})$.

\begin{tabular}{ccccc}
\hline Tratamentos & \multicolumn{3}{c}{ Epocas } & $\mathrm{E}_{3}$ \\
\cline { 2 - 5 } & $\mathrm{E}_{1}$ & $\mathrm{E}_{2}$ & $\mathrm{E}_{4}$ \\
\hline $\mathrm{T}_{0}$ & 68,56 & 61,61 & 61,36 & 50,21 \\
$\mathrm{~T}_{1}$ & 65,65 & 70,32 & 68,45 & 73,35 \\
$\mathrm{~T}_{2}$ & 67,36 & 69,76 & 67,19 & 69,15 \\
$\mathrm{~T}_{3}$ & 70,72 & 58,55 & 64,05 & 62,85 \\
$\mathrm{~T}_{4}$ & 66,58 & 51,20 & 58,65 & 55,31 \\
$\mathrm{~T}_{5}$ & 66,32 & 59,42 & 70,01 & 69,04 \\
$\mathrm{~T}_{6}$ & 66,53 & 68,24 & 68,56 & 67,68 \\
$\mathrm{~T}_{7}$ & 68,23 & 73,28 & 69,16 & 66,85 \\
\hline d.m.s. $-5 \%$ (Tukey) & 12,07 & 12,31 & 11,48 & 11,47 \\
\hline C.V. (\%) & & & & 9,62 \\
\hline
\end{tabular}

Observou-se que, de um modo geral, não houve diferenças significativas entre os efeitos dos tratamentos sobre a germinação. Apenas a testemunha foi inferior à menor dosagem de Panoctine $\left(\mathrm{T}_{2}\right)$, na primeira época $\left(\mathrm{E}_{1}\right)$, e ao tratamento com Arasan $\left(\mathrm{T}_{1}\right)$, na quarta época $\left(E_{4}\right)$.

Esses resultados, em parte, são semelhantes aos obtidos por ABRAHÃO \& ANDRADE (1961), que não verificaram diferença entre a germinação de sementes tratadas com PCNB, nas doses de 300 e $600 \mathrm{~g} / 100 \mathrm{~kg}$ e a testemunha. Por outro lado, esses autores relataram efeitos negativos de Arasan, o que não foi constatado no presente trabalho.

Por outro lado, os resultados encontrados no Quadro 3 mostraram que o vigor das sementes foi influenciado pelos tratamentos. Assim, o vigor das tratadas, com exceção para a maior dosagem de Panoctine $\left(\mathrm{T}_{4}\right)$, foi superior ao da testemunha, na terceira e quarta épocas $\left(E_{3}\right.$ e $\left.E_{4}\right)$.

Dentre os fungicidas utilizados, verificou-se que na segunda e quarta épocas $\left(E_{2}\right.$ e $\left.\mathrm{E}_{4}\right)$, as sementes submetidas à maior dose de Panoctine $\left(\mathrm{T}_{4}\right)$, mostraram-se menos vigorosas que as tratadas com Arasan $\left(\mathrm{T}_{1}\right)$, com Terracoat $\left(\mathrm{T}_{5}, \mathrm{~T}_{6}\right.$ e $\left.\mathrm{T}_{7}\right)$ e com a menor dose de Panoctine $\left(\mathrm{T}_{2}\right)$.

Esses resultados parecem confirmar as observações de CHRISTIDIS (1947), segundo as quais os resultados obtidos com o tratamento de sementes são muito influenciados pelas condições das sementes e pelo ambiente. Na presente pesquisa, o vigor das sementes tratadas foi superior ao da testemunha apenas na terceira e quarta épocas, quando, apesar de armazenadas em câmara seca as sementes já não apresentavam a mesma qualidade inicial.

2. Arroz: Germinação e Vigor - Os valores médios dos efeitos dos tratamentos, referentes às cinco épocas de germinação e de vigor, acham-se os Quadros 4 e 5 , respectivamente. 
QUADRO 4 - Germinaçáo de sementes de arroz: médias obtidas para os efeitos de tratamentos $(x=\operatorname{arcsen} \sqrt{\%})$.

\begin{tabular}{cccccc}
\hline Tratamentos & \multicolumn{5}{c}{ Epocas } \\
\cline { 2 - 6 } & $\mathrm{E}_{\mathbf{1}}$ & $\mathrm{E}_{\mathbf{2}}$ & $\mathrm{E}_{\mathbf{3}}$ & $\mathrm{E}_{\mathbf{4}}$ & $\mathrm{E}_{\mathbf{5}}$ \\
\hline $\mathrm{T}_{\mathbf{0}}$ & 68,50 & 75,90 & 74,09 & 78,94 & 67,36 \\
$\mathrm{~T}_{\mathbf{1}}$ & 77,29 & 79,35 & 78,09 & 76,26 & 77,43 \\
$\mathrm{~T}_{2}$ & 80,40 & 73,72 & 79,50 & 76,86 & 74,85 \\
$\mathrm{~T}_{3}$ & 76,55 & 77,43 & 71,69 & 77,43 & 72,87 \\
$\mathrm{~T}_{4}$ & 76,76 & 73,33 & 75,70 & 78,24 & 75,41 \\
$\mathrm{~T}_{5}$ & 79,50 & 76,36 & 76,20 & 76,77 & 73,73 \\
$\mathrm{~T}_{6}$ & 80,31 & 77,23 & 73,75 & 73,12 & 75,14 \\
$\mathrm{~T}_{7}$ & 73,23 & 73,87 & 74,85 & 75,26 & 75,91 \\
\hline d.m.s. - 5\% (Tukey) & 18,10 & 11,92 & 9,59 & 11,58 & 7,91 \\
\hline C.V. (\%) & 10,11 & 6,72 & 5,43 & 6,46 & 4,57 \\
\hline
\end{tabular}

QUADRO 5 - Vigor de sementes de arroz: médias obtidas para os efeitos de tratamentos $(x=\operatorname{arcsen} \sqrt{\%})$.

\begin{tabular}{cccccc}
\hline \multirow{2}{*}{ Tratamentos } & \multicolumn{5}{c}{ Épocas } \\
\cline { 2 - 6 } & $\mathrm{E}_{1}$ & $\mathrm{E}_{2}$ & $\mathrm{E}_{3}$ & $\mathrm{E}_{4}$ & $\mathrm{E}_{5}$ \\
\hline $\mathrm{T}_{\mathbf{0}}$ & 52,06 & 41,54 & 50,55 & 64,35 & 53,00 \\
$\mathrm{~T}_{1}$ & 52,27 & 47,58 & 45,00 & 66,36 & 59,58 \\
$\mathrm{~T}_{2}$ & 15,86 & 44,14 & 34,93 & 52,04 & 43,27 \\
$\mathrm{~T}_{3}$ & 17,43 & 24,95 & 24,32 & 44,42 & 36,74 \\
$\mathrm{~T}_{4}$ & 8,13 & 11,91 & 5,74 & 20,56 & 35,30 \\
$\mathrm{~T}_{5}$ & 48,21 & 43,56 & 50,78 & 66,91 & 47,59 \\
$\mathrm{~T}_{6}$ & 41,55 & 41,83 & 43,27 & 59,77 & 45,14 \\
$\mathrm{~T}_{7}$ & 32,79 & 38,43 & 44,13 & 62,01 & 46,43 \\
\hline d.m.s. - 5\% (Tukey) & 8,82 & 8,99 & 10,07 & 10,20 & 8,76 \\
\hline C.V. (\%) & 11,25 & 10,46 & 11,54 & 7,82 & 8,21 \\
\hline
\end{tabular}

Apenas na quinta época $\left(E_{5}\right)$ houve diferença significativa de germinação: a da testemunha $\left(T_{0}\right)$ foi inferior à das tratadas com Arasan $\left(T_{1}\right)$ e com as maiores doses de Panoctine e de Terracoat $\left(\mathrm{T}_{4}\right.$ e $\mathrm{T}_{7}$, respectivamente). Estes resultados diferem dos obtidos por OLIVEIRA \& FALCÃO (1970), pois esses autores não constataram diferença entre a germinação de sementes tratadas com Arasan e a testemunha. Por outro lado, os efeitos do tratamento com Terracoat foram semelhantes aos verificados por TOLEDO e outros (1971). 
De acordo com os resultados do Quadro 5, o vigor foi prejudicado pelo tratamento com Panoctine;' as médias referentes às três dosagens utilizadas $\left(T_{2}, T_{3}\right.$ e $\left.T_{4}\right)$, em quatro épocas, foram inferiores às da testemunha. Observou-se que, de um modo geral, o vigor das sementes tratadas com esse produto foi inversamente proporcional à dosagem empregada.

Por outro lado, não houve diferenças significativas entre a testemunha $\left(\mathrm{T}_{0}\right)$, Ara$\operatorname{san}\left(\mathrm{T}_{1}\right)$ e as três doses de Terracoat $\left(\mathrm{T}_{5}, \mathrm{~T}_{6}\right.$ e $\left.\mathrm{T}_{7}\right)$.

Examinando-se os resultados dos testes de vigor e de germinaçâo, verifica-se que os mesmos não são comparáveis; de uma maneira geral, os tratamentos fungicidas não influenciaram a germinação, enquanto os testes de vigor foram mais rigorosos na avaliação dos efeitos dos tratamentos, justificando sua utilização no presente trabalho.

3. Soja: Germinação e Vigor - Nos Quadros 6 e 7 são apresentadas as médias referentes aos testes de germinação e vigor.

QUADRO 6 - Germinação de sementes de soja: médias obtidas para os efeitos de tratamentos $(\mathrm{x}=\operatorname{arcsen} \sqrt{\%})$.

\begin{tabular}{cccccc}
\hline Tratamentos & \multicolumn{5}{c}{ Epocas } \\
\cline { 2 - 6 } & $\mathrm{E}_{1}$ & $\mathrm{E}_{2}$ & $\mathrm{E}_{3}$ & $\mathrm{E}_{4}$ & $\mathrm{E}_{5}$ \\
\hline $\mathrm{T}_{\mathbf{0}}$ & 83,05 & 83,90 & 78,69 & 62,62 & 69,33 \\
$\mathrm{~T}_{1}$ & 83,35 & 87,96 & 80,16 & 76,26 & 75,70 \\
$\mathrm{~T}_{2}$ & 83,57 & 86,45 & 78,44 & 71,78 & 74,55 \\
$\mathrm{~T}_{3}$ & 77,38 & 79,50 & 80,16 & 66,18 & 73,12 \\
$\mathrm{~T}_{4}$ & 83,05 & 80,02 & 77,58 & 69,94 & 73,23 \\
$\mathrm{~T}_{5}$ & 85,08 & 85,08 & 80,87 & 73,90 & 74,13 \\
$\mathrm{~T}_{6}$ & 90,00 & 82,19 & 85,08 & 69,50 & 79,50 \\
$\mathrm{~T}_{7}$ & 85,93 & 87,96 & 81,01 & 73,24 & 81,53 \\
\hline d.m.s. $-5 \%$ (Tukey) & 13,57 & 12,22 & 10,12 & 11,02 & 9,46 \\
\hline C.V. (\%) & 6,94 & 6,21 & 5,48 & 6,63 & 5,39 \\
\hline
\end{tabular}


QUADRO 7 - Vigor de sementes de soja: médias obtidas para os efeitos de tratamentos $(\mathrm{x}=\operatorname{arcsen} \sqrt{\%})$.

\begin{tabular}{cccccc}
\hline Tratamentos & \multicolumn{5}{c}{ Épocas } \\
\cline { 2 - 6 } & $\mathrm{E}_{\mathbf{1}}$ & $\mathrm{E}_{\mathbf{2}}$ & $\mathrm{E}_{\mathbf{3}}$ & $\mathrm{E}_{\mathbf{4}}$ & $\mathrm{E}_{\mathbf{5}}$ \\
\hline $\mathrm{T}_{\mathbf{0}}$ & 51,08 & 61,86 & 63,46 & 63,00 & 62,42 \\
$\mathrm{~T}_{\mathbf{1}}$ & 54,29 & 67,92 & 69,32 & 67,37 & 67,36 \\
$\mathrm{~T}_{\mathbf{2}}$ & 53,74 & 59,72 & 71,18 & 63,86 & 66,06 \\
$\mathrm{~T}_{\mathbf{3}}$ & 40,67 & 49,05 & 66,21 & 53,14 & 58,10 \\
$\mathrm{~T}_{\mathbf{4}}$ & 34,41 & 41,47 & 65,34 & 53,00 & 62,08 \\
$\mathrm{~T}_{5}$ & 55,69 & 58,34 & 65,37 & 67,14 & 58,72 \\
$\mathrm{~T}_{6}$ & 53,50 & 66,33 & 67,40 & 63,45 & 65,72 \\
$\mathrm{~T}_{7}$ & 50,47 & 64,60 & 71,54 & 68,80 & 64,90 \\
\hline d.m.s. - 5\% (Tukey) & 15,76 & 14,40 & 9,95 & 9,39 & 8,84 \\
\hline C.V. (\%) & 13,69 & 10,50 & 6,30 & 6,39 & 5,99 \\
\hline
\end{tabular}

Os tratamentos empregados não exerceram influência marcante sobre a germinação da soja. Apenas na quinta época, as sementes submetidas às maiores dosagens de Terracoat $\left(T_{6}\right.$ e $\left.T_{7}\right)$ apresentaram germinação superior à da testemunha.

Essas observações não condizem com as de NETO ë colaboradores (1975); segundo esses autores, os tratamentos com Panoctine $\left(200 \mathrm{~cm}^{3} / 100 \mathrm{~kg}\right)$ e Terracoat $\left(200 \mathrm{~cm}^{3} /\right.$ $/ 100 \mathrm{~kg}$ ) forarn prejudiciais à germinação, o que não foi verificado no presente estudo. Além de variações nas metodologias adotadas, as características fisiológicas das sementes empregadas devem ter influido nos diferentes resultiados obtidos.

No presente trabalho, não foram constatados efeitos benéficos do fungicida Arasan sobre a germinação, conforme relataram PEREIRA GONÇALO (1963) e NETO e colaboradores (1975).

Por outro lado, os testes de vigor não acusaram diferenças entre Arasan $\left(T_{1}\right)$, as três dosagens de Terracoat $\left(\mathrm{T}_{5}, \mathrm{~T}_{6}\right.$ e $\left.\mathrm{T}_{7}\right)$ e a testemunha. A maior dosagem de Panoctine $\left(\mathrm{T}_{4}\right)$ prejudicou significativamente o vigor das sementes, segundo as médias obtidas na primeira, segunda e quarta épocas $\left(E_{1}, E_{2}\right.$ e $\left.E_{4}\right)$.

O estudo dos resultados obtidos com as sementes de soja, tal como com as de algodão e de arroz, evidenciou a maior eficiência do teste de vigor para diferenciar níveis de qualidade entre as sementes tratadas e a testemunha. Os testes de vigor forneceram resultados mais consistentes, apontando diferenças não mostradas pelos testes de germinação, que se repetiram nas diferentes épocas. 


\section{CONCLUSÕES}

As análises dos dados e a interpretação dos resultados do presente trabalho permitiram concluir que, dos produtos testados, Panoctine foi o único prejudicial à qualidade das sementes: as três dosagens mostraram-se fitotóxicas para arroz, enquanto a de $600 \mathrm{~cm}^{3} / 100 \mathrm{~kg}$ prejudicou o vigor das semertes de algodão e de soja.

A aplicação de Arasan e das três dosagens de Terracoat destacaram-se pelos seus efeitos benéficos sobre o vigor de sementes de algodão e não prejudiciais ao de soja e de arroz.

Por outro lado, recomenda-se o emprego de testes de vigor em pesquisas desta natureza, para a obtenção de resultados mais seguros.

\section{SUMMARY}

\section{EFFECTS OF NON-MERCURIAL FUNGICIDES ON THE GERMINATION AND VIGOR OF COTTON, RICE AND SOYBEAN SEEDS}

This research was carried out with the main objective of studying the performance of cotton, rice and soybean seeds when treated with non-mercurial fungicides, by means of germination and vigor tests.

The fungicides used were: Arasan (Thiram 75\% i.a.), Panoctine (Guazatine 35\% i.a.) and Terracoat (23.2\% PCNB $+5,8 \%$ Terrazole). The dosage of the first product was $100 \mathrm{~g} / 100 \mathrm{~kg}$ of seeds, whereas for the others two were 200,400 and $600 \mathrm{~cm}^{3}$ of the commercial product $/ 100 \mathrm{~kg}$ of seeds were utilized.

Under the conditions of the experiment, the three dosages of Panoctine were toxic for rice seeds and the highest dosage of this product $\left(600 \mathrm{~cm}^{3} / 100 \mathrm{~kg}\right)$ lowered cotton and soybean seed vigor.

Arasan and Terracoat had no effect on germination and vigor of rice and soybean but had a beneficial effect on cotton seed vigor. 


\section{LITERATURA GITADA}

ABRAHÃO, J. \& ANDRADE, A.C., 1961. Experiência de desinfecção de sementes de algodão no período de 1955/59. Arq. Inst. Biol., 28 :133-148.

ABRAHĀO, J., BASTOS CRUZ, B.P. \& GREGORI, R., 1964. Tratamento das sementes de algodão como meảida de controle das doenças das sementeiras. O Biológico, 30(7):169-173.

ATHOW, R. \& CALDWELL, R.P.: 1956. The influence of seed treatment and planting rate on the emergence and yield of soybeans. Phytopathology, 4691-95.

CHRISTIDIS, B.G., 194\%. Cotton seed treatment for controlling seedling diseases. Cotton Res. Inst. Sci. Bull., 1:1-32.

DELOUCHE, J.C. \& CALDWELL, W.P., 1960. Seed vigor and vigor tests. Proc. Assoc. Off. Seed. Anal., 50:124-129.

MELLO, R.E.T. \& ROSSETTI, V., 1960. Observações sobre a brusone do arroz e seu controle. O Biológico, 26(1):218-222.

MINISTÉRIO DA AGRICULTURA, 1967. Regras para Análise de Sementes. Escritório da Produção Vegetal. Equipe Técnica de Sementes e Mudas. 120 pág.

NETO, N., BESKOW, G., BONETTI, L.P. \& MIRANDA, T.R., 1975. Efeito do tratamento de sementes com fungicidas e sua influência sobre o rendimento e algumas características da soja. III Reunião Conjunta de Pesquisa da Soja RS/SC - Contribuição da FECOTRIGO: 95-99.

OLIVEIRA, S.A. \& FALCÃO, L.A., 1970. Proteção de sementes de arroz com fungicidas. An. III Sem. Bras. Sementes, Recife, PE: 282-284.

PEREIRA GONÇALO, J., 1963. Informações preliminares sobre tratamento de sementes de soja com fungicidas e sua influência sobre o poder germinativo, em condições de laboratório. An. IV Sem. Panam. Sementes, Brasil: 196.

PULIDO, M.L., 1972. Un nuevo tratamiento efectivo de semillas para reemplazar a productos mercuriales. An. VII Sem. Panam. Semillas, México: 18-27.

TOLEDO, A.C.D., AMARAL, R.E.M., SOUZA, D.M. \& ARRUDA, H.V., 1971. Fungicidas sucedâneos dos mercuriais para tratamento de sementes de arroz. O Biológico, 37(11):300-302. 
